This item was submitted to Loughborough's Research Repository by the author.

Items in Figshare are protected by copyright, with all rights reserved, unless otherwise indicated.

\title{
Phase-field crystal modeling of heteroepitaxy and exotic modes of crystal nucleation
}

PLEASE CITE THE PUBLISHED VERSION

https://doi.org/10.1016/j.jcrysgro.2016.06.056

PUBLISHER

(c) Elsevier

VERSION

AM (Accepted Manuscript)

\section{PUBLISHER STATEMENT}

This work is made available according to the conditions of the Creative Commons Attribution-NonCommercialNoDerivatives 4.0 International (CC BY-NC-ND 4.0) licence. Full details of this licence are available at: https://creativecommons.org/licenses/by-nc-nd/4.0/

\section{LICENCE}

CC BY-NC-ND 4.0

\section{REPOSITORY RECORD}

Podmaniczky, Frigyes, Gyula I. Toth, Gyorgy Tegze, Tamas Pusztai, and Laszlo Granasy. 2019. "Phase-field Crystal Modeling of Heteroepitaxy and Exotic Modes of Crystal Nucleation". figshare. https://hdl.handle.net/2134/27267. 


\title{
Phase-field crystal modeling of heteroepitaxy and exotic modes of crystal nucleation
}

\author{
Frigyes Podmaniczky, ${ }^{1}$ Gyula I. Tóth, ${ }^{1,2}$ György Tegze, ${ }^{1}$ Tamás Pusztai, ${ }^{1}$ and László Gránásy ${ }^{1,3, \S}$ \\ ${ }^{1}$ Institute for Solid State Physics and Optics, Wigner Research Centre for Physics, P O Box 49, H-1525 Budapest, Hungary \\ ${ }^{2}$ Department of Physics and Technology, University of Bergen, Allégaten 55, N-5007 Bergen, Norway \\ ${ }^{3}$ BCAST, Brunel University, Uxbridge, Middlesex, UB8 3PH, UK \\ $\S$ Corresponding authors: granasy.laszlo@wigner.mta.hu and podmaniczky.frigyes@wigner.mta.hu
}

We review recent advances made in modeling heteroepitaxy, two-step nucleation, and nucleation at the growth front within the framework of a simple dynamical density functional theory, the Phase-Field Crystal (PFC) model. The crystalline substrate is represented by spatially confined periodic potentials. We investigate the misfit dependence of the critical thickness in the StranskiKrastanov growth mode in isothermal studies. Apparently, the simulation results for stress release via the misfit dislocations fit better to the People-Bean model than to the one by Matthews and Blakeslee. Next, we investigate structural aspects of two-step crystal nucleation at high undercoolings, where an amorphous precursor forms in the first stage. Finally, we present results for the formation of new grains at the solid-liquid interface at high supersaturations / supercoolings, a phenomenon termed Growth Front Nucleation (GFN). Results obtained with diffusive dynamics (applicable to colloids) and with a hydrodynamic extension of the PFC theory (HPFC, developed for simple liquids) will be compared. The HPFC simulations indicate two possible mechanisms for GFN.

Keywords: A1: Solidification, Heterogeneous nucleation, Heteroepitaxy, Growth Front Nucleation, Phase-Field Crystal model, Hydrodynamic theory of crystallization

\section{Introduction}

Under practical conditions, the crystallization of supersaturated/supercooled liquids starts by either heteroepitaxial growth or heterogeneous nucleation on foreign surfaces (including container walls or foreign particles). The microscopic description of these phenomena requires an atomistic approach. A recently developed theoretical tool, suitable for such purposes, is a simple dynamical density functional theory of classical particles, termed the Phase-Field Crystal (PFC) model [14], which has previously been applied to address crystal nucleation [4-8] and growth under various conditions $[2-4,9,10]$. This model incorporates several crystalline phases (bcc, hcp, and fcc [10]), besides the liquid and the glass [11, 12]. In the original version of the theory [1-11], the time evolution of the system is described by overdamped conservative (diffusive) dynamics, which is appropriate for modeling colloidal crystalline aggregation. An advantage of this model is that it can address freezing on a diffusive time scale [1], and can handle millions of particles relatively easily [4]. To address the crystallization of normal fluids, a hydrodynamic theory based on the PFC model has been put forward [13], which we denote here as HPFC.

The PFC model has already contributed to a better understanding of phenomena associated with the crystallization of undecooled/supersaturated liquids induced by solid surfaces:

(a) This model was used to model heteroepitaxy on foreign crystal surfaces, and a good agreement with the theory of Matthews and Blakeslee [14] has been reported for 2D hexagonal crystals grown on a hexagonal crystal of varied lattice constant $[1,2,15]$. Similar results were obtained for a square lattice substrate [16]. Heterogeneous nucleation was studied on unstructured substrates $[4,17]$ and substrates represented by a periodic potential term in the free energy functional $[4,7,8,16]$. It has been shown that neither the nucleation barrier, nor the contact angle are monotonous functions of the lattice mismatch $[4,7$, 8].

(b) In agreement with theory [18], molecular dynamics simulations [19], and colloid experiments [20-22], recent investigation relying on the PFC model have shown that at large supersaturations/undercoolings crystal nucleation is a two-step process, in which an amorphous precursor assists the formation of the crystalline phase $[4,6,8]$.

(c) Finally, earlier efforts relying on the original PFC model have shown that the creation of new grains at a propagating front, a phenomenon known as Growth Front Nucleation $[23,24]$, was only possible at supersaturations beyond the stability limit of the liquid phase [25]. Unfortunately, in the presence of fluctuations this phenomenon cannot be realized, as crystallization starts everywhere in the presence of noise; i.e., the formation of new grains at the front is suppressed by the copious nucleation and crystallization taking place in the whole liquid volume.

In this paper, we present recent advances made in PFC modeling in the following three areas:

(i) Prediction of critical thickness as a function of misfit in isothermal treatments within the PFC model;

(ii) The structural aspects of two-step homogeneous crystal nucleation via amorphous precursor in the PFC model;

(iii) Modeling of growth front nucleation within the HPFC model.

\section{The PFC model}

In the PFC approach, the local state of matter is characterized by the time-averaged particle density, $\rho$. The dimensionless free energy of the inhomogeneous system taken relative to a homogeneous reference fluid (of density $\rho_{\mathrm{L}, \mathrm{ref}}$ ) reads as 


$$
\Delta F=\int d \mathbf{r}\left\{\frac{\psi}{2}\left[-\varepsilon+\left(1+\nabla^{2}\right)^{2}\right] \psi+\frac{\psi^{4}}{4}\right\},
$$

where $\psi \propto\left(\rho-\rho_{\mathrm{L}, \text { ref }}\right) / \rho_{\mathrm{L} \text {,ref }}$ is the scaled density difference. The reduced temperature $\varepsilon$ can be connected to such physical properties as the bulk moduli of the fluid and crystalline phases at the reference density and temperature. Eq. (1) can be deduced $[2,26]$ from the perturbative density functional theory of Ramakrishnan and Yussouff [27].

\subsection{The Euler-Lagrange equation}

The solutions that extremize the free energy functional can be obtained by solving the Euler-Lagrange equation (ELE) [9]

$$
\frac{\delta \Delta F}{\delta \psi}=\left.\frac{\delta \Delta F}{\delta \psi}\right|_{\psi_{0}},
$$

where $\psi_{0}$ is the reduced particle density of the reference state, while periodic boundary condition is assumed at the borders. Inserting the free energy functional into Eq. (2) and rearranging the terms, one obtains

$$
\left[-\varepsilon+\left(1+\nabla^{2}\right)^{2}\right]\left(\psi-\psi_{0}\right)=-\left(\psi^{3}-\psi_{0}^{3}\right) .
$$

\subsection{The diffusive equation of motion}

In the original PFC model $[1-11,13,16]$ the time evolution of the reduced number density is described using conserved overdamped dynamics realized by the following dimensionless equation of motion (EOM):

$$
\frac{\partial \psi}{\partial t}=\nabla \cdot\left\{\nabla \frac{\delta \Delta F}{\delta \psi}+\xi_{j}\right\}
$$

where $\delta \Delta F[\psi] / \delta \psi$ denotes the functional derivative of the free energy $F$ with respect to the scaled number density difference, whereas $\xi_{\mathrm{j}}$ is a colored Gaussian flux noise of correlator $\left\langle\xi_{\mathrm{j}}(\mathbf{r}, t)\right.$, $\left.\xi_{\mathrm{j}}\left(\mathbf{r}^{\prime} t^{\prime}\right)\right\rangle=-\alpha^{2} \nabla^{2} g\left(\left|\mathbf{r}-\mathbf{r}^{\prime}\right|, \sigma_{0}\right) \delta\left(t-t^{\prime}\right), \alpha$ the noise strength, and $g\left(\left|\mathbf{r}-\mathbf{r}^{\prime}\right|, \sigma_{0}\right)$ a high frequency cutoff function [28], for wavelengths shorter than the interatomic spacing $\left(\sigma_{0}\right)$.

\subsection{Hydrodynamic theory of solidification}

In developing the hydrodynamic model of crystalline solidification our starting point was fluctuating nonlinear hydrodynamics as formulated in [29]. Accordingly, the momentum transport and continuity equations are written in the form

$$
\begin{aligned}
& \frac{\partial \mathbf{p}}{\partial t}+\nabla \cdot(\mathbf{v} \otimes \mathbf{p})=\nabla \cdot[\mathbf{R}(\rho)+\mathbf{D}(\mathbf{v})+\mathbf{S}] \\
& \frac{\partial \rho}{\partial t}+\nabla \cdot \mathbf{p}=0
\end{aligned}
$$

where $\mathbf{p}(\mathbf{r}, t)$ is the momentum, $\rho(\mathbf{r}, t)$ the mass density, $\mathbf{v}=$ $\mathbf{p} / \rho$ the velocity, whereas $\nabla \cdot \mathbf{R}=-\rho \nabla\{\delta \Delta F[\rho] / \delta \rho\} \approx$ $-\rho_{0} \nabla\{\delta \Delta F[\rho] / \delta \rho\}$ is the reversible stress tensor, $\rho_{0}$ a reference density, and $\mathbf{D}=\mu_{\mathbf{S}}\left\{(\mathbf{v} \otimes \mathbf{p})+(\mathbf{v} \otimes \mathbf{p})^{\mathrm{T}}\right\}+\left[\mu_{\mathrm{B}}-(2 / 3) \mu_{\mathrm{S}}\right](\nabla \cdot \mathbf{v})$ the dissipative stress tensor, while $\mathbf{S}$ is a stochastic momentum noise of correlator

$$
\begin{aligned}
& \left\langle S_{i j}(\mathbf{r}, t), S_{k l}\left(\mathbf{r}^{\prime}, t^{\prime}\right)\right\rangle=\left(2 k T \mu_{S}\right) \times \\
& {\left[\left(\delta_{i k} \delta_{j l}-\delta_{j k} \delta_{i l}\right)+\left(\frac{\mu_{B}}{\mu_{S}}-\frac{2}{3}\right) \delta_{i j} \delta_{k l}\right] \delta\left(\mathbf{r}-\mathbf{r}^{\prime}\right) \delta\left(t-t^{\prime}\right) .}
\end{aligned}
$$

Here $\mu_{\mathrm{s}}$ and $\mu_{\mathrm{B}}$ are the shear and bulk viscosities.
To avoid violent interatomic flow in the bulk crystal due to the enormous density gradients in the hydrodynamic equations, we apply coarse-grained momentum and density in computing the velocity: $\mathbf{v}=\hat{\boldsymbol{p}} / \hat{\rho}$. (For details see Ref. [13].) The HPFC model recovers the proper dispersion relation for long wavelength acoustic phonons, a steady state front velocity that is inversely proportional to the viscosity, and describes stress relaxation reasonably.

\subsection{Numerical solutions}

The ELE has been solved numerically in $2 \mathrm{D}$ and 3D, using a semi-spectral successive approximation scheme combined with the operator-splitting method $[9,30]$. The EOM and the hydrodynamic equations have been solved using a semi-implicit spectral scheme based on operator splitting [30]. The codes were run on $35 \mathrm{GPU}$ cards of various types.

\section{Results and discussion}

\subsection{Critical thickness in heteroepitaxy}

The advance of heteroepitaxial growth is shown in Fig. 1, which displays the Voronoi polyhedral analysis. Particles with 4, 5, 6, and 7 neighbors are colored grey, blue, yellow, and red. Accordingly, the dislocations are represented by red-blue pairs. As expected from theory, below a critical thickness depending on the magnitude of the misfit, $f=\left(\sigma_{0}-\sigma\right) / \sigma$, laterally stretched or compressed crystal grows. (Here $\sigma_{0}$ and $\sigma$ are the lattice constants of the growing crystal and the substrate, respectively.) Beyond the critical thickness, the Asaro-Tiller-Grinfeld (ATG) instability [31-33] sets in, due to the stress present in the growing crystal, which thus yields surface buckling (Fig. 1, see also $[1,2,15,33])$. In the cusps of the surface, misfit dislocations nucleate that release stress. Unlike a previous study [16], where growth during cooling was investigated, here we model heteroepitaxial growth under isothermal conditions. In the present simulations, 2D hexagonal crystal grows on the surface of a square substrate, whose lattice constant is varied. The reduced temperature was chosen as $\varepsilon=0.18$, whereas three noise strengths were applied: $\alpha=0,10^{-4}$, and $10^{-2}$. The respective critical thickness $\left(h_{\mathrm{c}}\right)$ values are plotted vs. the lattice misfit in Fig. 2. It is found that, as expected, $h_{\mathrm{c}}$ diverges at zero misfit [Fig. 2(a)], and in agreement with previous results [16], $h_{\mathrm{c}}$ decreases with increasing noise strength. Plotting $[1+$ $\left.\ln \left(h_{\mathrm{d}} / b\right)\right] /\left(h_{\mathrm{d}} / b\right)$ with respect to $f$ should result in a straight line crossing the origin, provided that the results obey the Matthews-Blakeslee (MB) model [14]. Here $b$ is the length of the Burgers vector, which is $\sigma_{0}$ in our case. Assuming screw dislocations and a high dislocation density, a different scaling was proposed by People and Bean (PB) [34], where plotting $\left[A \cdot \ln \left(h_{\mathrm{c}} / b\right) /\left(h_{\mathrm{c}} a_{s} / b^{2}\right)\right]^{1 / 2}$ vs. $f$ is expected to produce a straight line, where $A=(16 \pi \sqrt{2})^{-1 / 2}$. Remarkably, the MB plots are rather curved for the present simulations [Fig. 2(b)], whereas the PB plots are nearly straight lines in the investigated regime [Fig. 2(c)], although to reach the origin, the plots still need to curve slightly. This suggests that the $h_{\mathrm{c}}(f)$ relationship emerging from the PFC simulations fits better to the behavior predicted by the PB model than the one by the MB model. We recall in this respect that some of the experiments are better described by the PB model, whereas others fit rather to the MB 

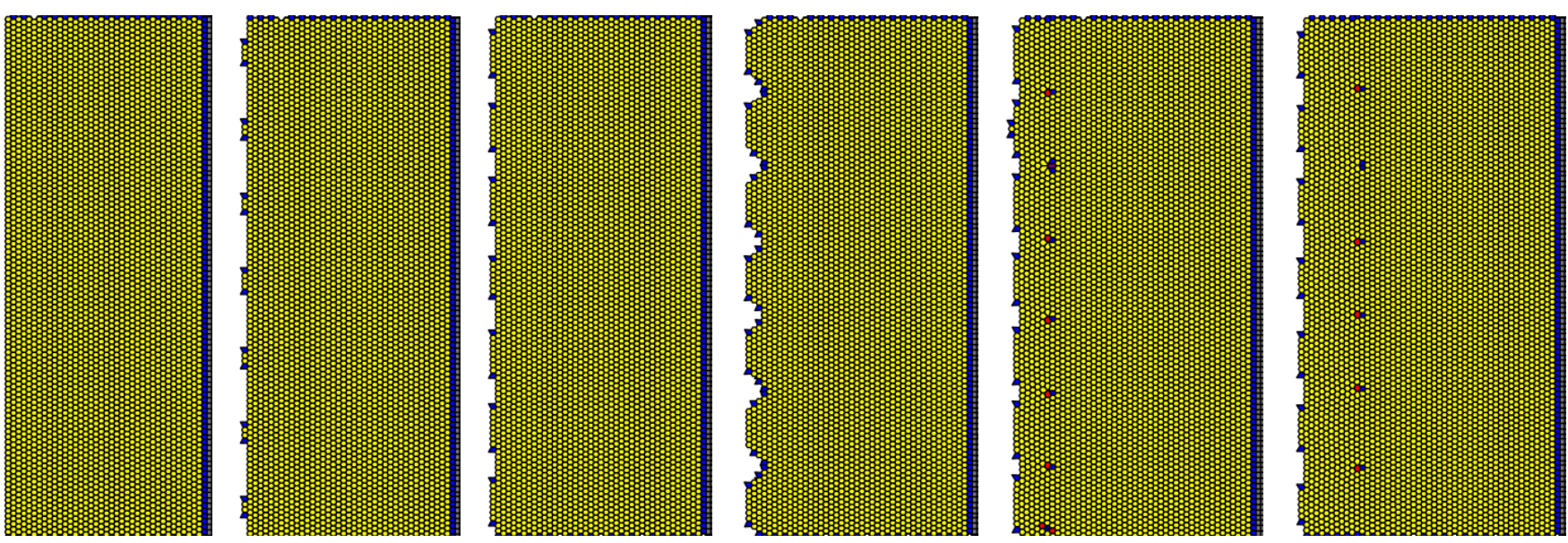

Fig. 1. Snapshots of heteroepitaxial growth of a triangular crystal in a PFC simulation at $\varepsilon=0.18, \psi 0=-0.25$, and $\alpha=0$, on a square-lattice substrate of $\sigma=0.92 \sigma 0$, taken at dimensionless times $t=2300,2400,2500,2600,2700$, and 3300, respectively. Results of the Voronoi polyhedral analysis is shown. Coloring: particles of 4, 5, 6, and 7 neighbors are painted grey, blue, yellow, and red, respectively; whereas the liquid is white. The substrate lies vertically on the RHS, and is composed of 4 coordinated particles (grey), with a monolayer of 5 coordinated particles (blue) on its surface. The free surface of the epitaxial layer is on the left. The misfit dislocations are represented by 5-7 pairs (blue-red). The vertical size of the simulation box is commensurate with both the substrate and the stress free bulk crystal $\left(L_{y}=92 \sigma 0=100 \sigma\right)$. Following the mechanism described in Refs. $[1,2,15,33]$, misfit dislocations appear in the depressions of the surface formed due to the ATG instability. In agreement with the findings of Ref. [33], the wavelength of buckling $\left(\lambda=13.14 \sigma_{0}\right)$ is larger than expected for the fully relaxed state $\left(\lambda_{R}=11.5 \sigma_{0}\right)$ : 7 waves form instead of 8 .

model [34]. However, the agreement with the PB model is usually attributed to the fact that the combined effects of finite experimental resolution with initially sluggish lattice relaxation
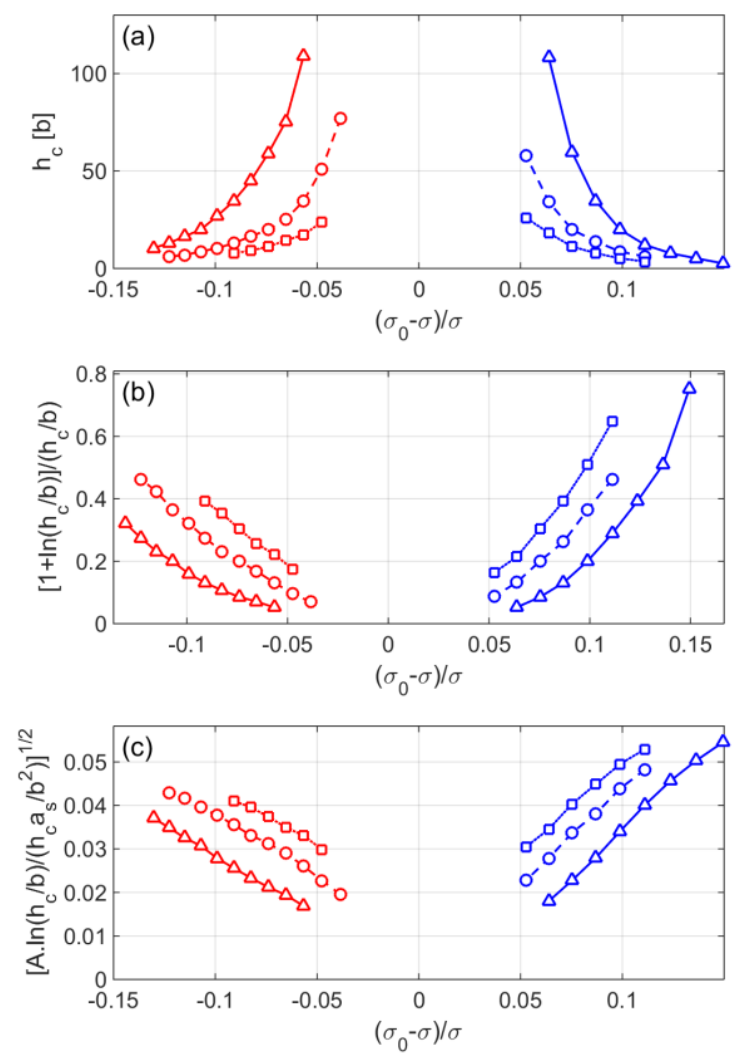

Fig. 2. Formation of misfit dislocations in $2 \mathrm{D}$ hexagonal crystal growing on a square lattice substrate as predicted by the PFC model: (a) Dependence of the critical thickness $\left(h_{\mathrm{c}}\right)$ on the misfit $(f)$ for reduced temperature $\varepsilon=0.18$, and noise strengths $\alpha=0,10^{-4}$, and $10^{-2}$ denoted by triangles, circles, and squares, respectively. (b) The Matthews-Blakeslee plot [13] for the same data. (c) The respective People-Bean plot [30]. With the present definition of the misfit, $f>0$ for compressive stress, and $f<0$ for tensile stress. can cause the experiments to overestimate the critical layer thickness [35]. Work is underway to compare the present results with other theoretical models, including that by Fischer $e t$ al. [36].

Remarkably, in the PFC simulations the magnitude of the slope of the plots depends on the sign of the misfit, as also seen in experiments [35]. Indeed for relatively small stresses $(|f|<\sim$ $7 \%), h_{\mathrm{c}}$ is larger for compressive stress than for tensile stress, whereas after a crossover at $|f| \sim 7 \%$, the opposite relationship is seen for large stresses.

\subsection{Structural aspects of two-step homogeneous nucleation in highly undercooled liquids.}

In the PFC model crystal nucleation takes place in two steps at high undercoolings or supersaturations: in the first step an amorphous precursor forms, and helps the appearance of the crystalline (bcc) phase $[6,8,10]$. Apparently, this phenomenon is highly relevant not only for colloids [18, 21, 22, 37], but for such simpler systems as the Lennard-Jones fluid [18] and the hard sphere system [19], raising the possibility that the presence of an amorphous precursor might be a general feature of crystal nucleation in highly undercooled or supersaturated liquids. In this subsection, we investigate the structural aspects of this process within the framework of the PFC model. In this, we rely on the bond order parameters $q_{\mathrm{i}}$ and $\bar{q}_{\mathrm{i}}$ introduced by Steinhardt et al. [38] and Lechner and Dellago [39], respectively of which $q_{\mathrm{i}}$ consider the first neighbor shell around the particles, whereas $\bar{q}_{\text {i }}$ take into account the relative orientations to atoms in the first and second neighbor shells.

The phenomenon of two-step nucleation is illustrated in Fig. 3 for instantaneous quenching of the homogeneous liquid from above the liquidus ( $\varepsilon=0.1336$ ) to $\varepsilon=0.1667$. The scaled density of the initial liquid was $\psi_{0}=-0.25$, while the noise strength was $\alpha=0.42$. In the upper row of Fig. 3, the particles that have bcc-like neighborhood are painted red, whereas the rest qualifies as amorphous (colored white). Here solidification 

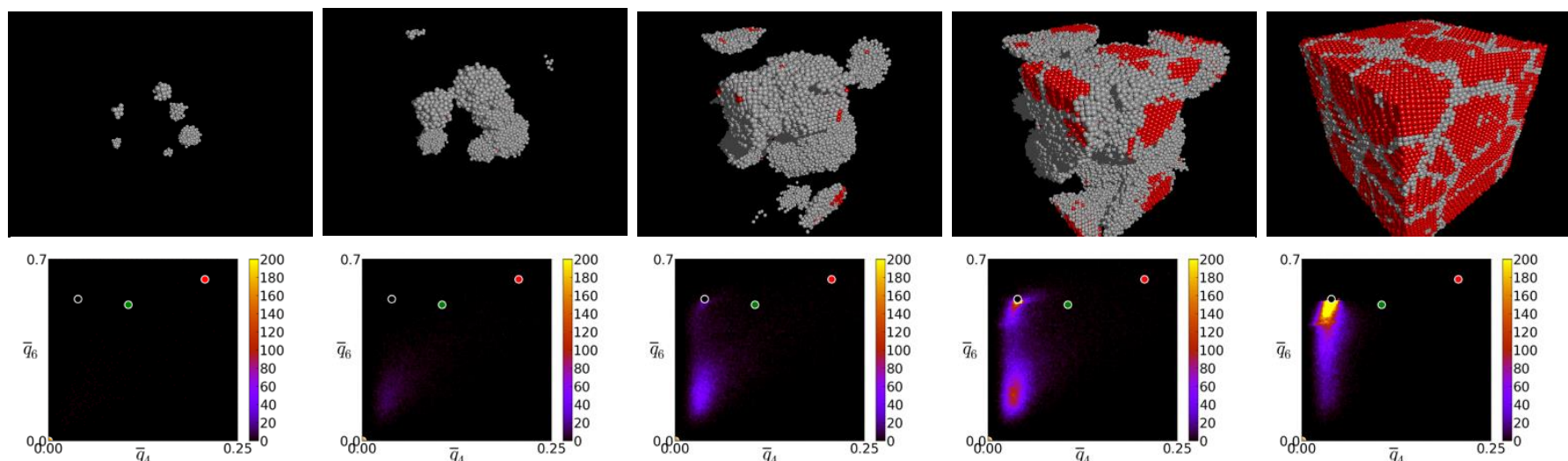

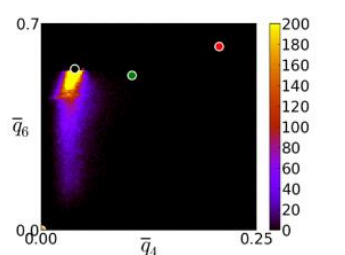

Fig. 3. Snapshots of two-step nucleation in the PFC model at $\varepsilon=0.1684$ and $\psi_{0}=-0.25$ at dimensionless times $t=50,70,90,110$, and 1000, respectively. Upper row: red (bbc-like neighborhood) $-q_{4} \in[0.02,0.07]$ and $q_{6} \in[0.48,0.52]$; white (amorphous) — the remaining particles. The liquid phase is transparent. Lower row: $q_{4}$ vs. $q_{6}$ bond-order parameter map (as defined by Lechner and Dellago [39]; these order parameters consider the first and second neighbors for the same configurations. (The circles painted yellow, black, green, and red indicate the positions of the ideal icosahedral, bcc, hcp, and fcc structures, respectively.) Solidification appears to start with the nucleation of amorphous domains.

(a)

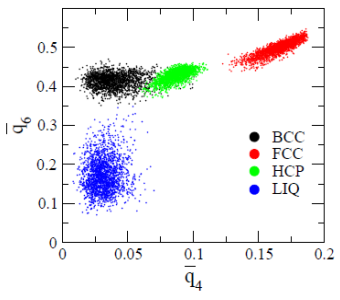

(b)

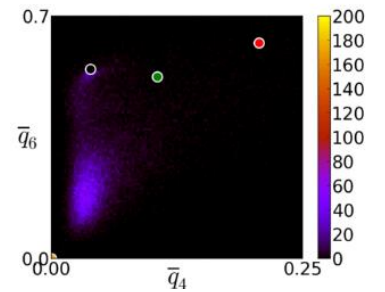

Fig. 4. Comparison of the $\bar{q}_{4}$ vs. $\bar{q}_{6}$ bond-order parameter maps obtained (a) for the Lennard-Jones system from molecular dynamics simulations (reproduced with permission from [39] (C) American Institute of Physics) and (b) for the solidifying PFC system (central panels of Fig. 1). Note the points for the ideal crystal structures in panel (b) fall higher than the ones obtained with thermal fluctuations in panel (a). Apparently, the structure of the amorphous precursor observed in the PFC model is close to the structure of the bulk liquid in the Lennard-Jones system. starts with the formation of amorphous clusters, and the bcc structure appears later. In the second row of Fig. 3, the respective $\bar{q}_{4}$ vs. $\bar{q}_{6}$ bond order parameter maps are shown together with the points corresponding to the ideal icosahedral, bcc, fcc, and hcp neighborhoods. Indeed, the first appearing solid is the amorphous precursor represented by the blue nebula positioned slightly above and right of the lower left corner, whose amplitude (the amount of the amorphous neighborhoods) first increases with time, but soon disappears as bcc crystallization starts, with some remnants remaining present at the grain boundaries. Interestingly, the structure of the amorphous precursor is close to that of the liquid in the Lennard-Jones system, as implied by the respective $\bar{q}_{4}$ vs. $\bar{q}_{6}$ maps (see Fig. 4 ).

Following the structural analysis employed in recent Brownian dynamics (BD) simulations [40] and colloid crystallization experiments [37], we performed a similar structural analysis for the amorphous (white) areas in Fig. 3. Analyzing BD simulations Kawasaki and Tanaka arrived to the conclusion that in the undercooled liquid the formation of a Medium Range Crystalline Order (MRCO) precedes crystal nucleation. MRCO is defined so that $0.28<\bar{q}_{6}<0.4$, while $\bar{q}_{6}<0.28$ qualifies as
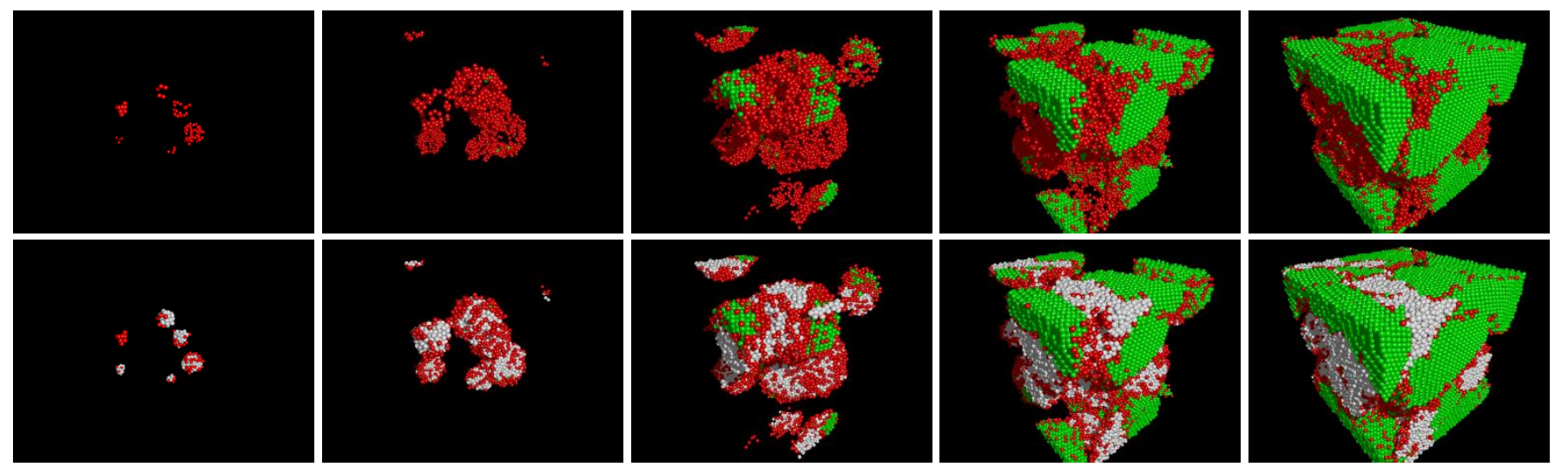

Fig. 5. Structural analysis of two-step nucleation observed in the PFC simulation displayed in Fig. 1. The coloring scheme proposed by Tan et. al [37] has been used. (Here the rightmost panel corresponds to dimensionless time $t=130$.) Upper row: only MRCO and bcc crystal are shown. Bottom row: amorphous, MRCO, and bcc domains are displayed. [Coloring: green (bcc crystal) $-\bar{q}_{6} \geq 0.27$ and $\xi \geq 7$, where $\xi$ is the number of solid bonds; red (MRCO = Medium Range Crystalline Order) $-\bar{q}_{6} \geq 027$ and $\left.\xi<7\right]$; white (amorphous) $-\bar{q}_{6}<0.27$.] Apparently, MRCO and the amorphous precursor appear roughly parallel (see Fig. 6), whereas the bulk bcc structure forms later in contact with the MRCO domains. It seems that the MRCO domains evolve on the surface of amorphous domains, indicating a process akin to heterogeneous nucleation. 


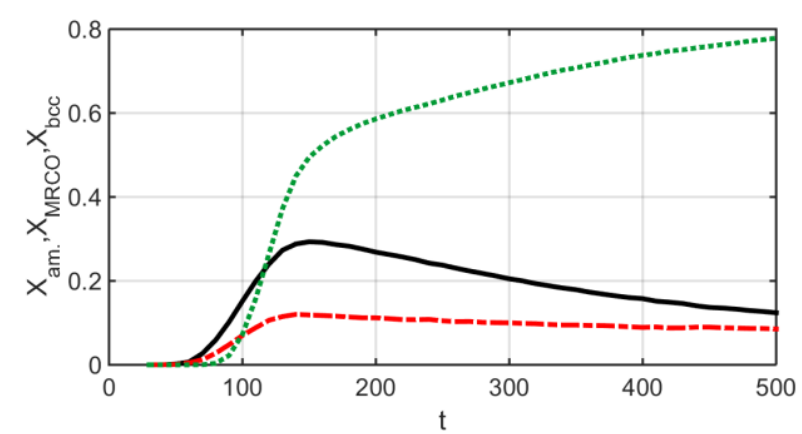

Fig. 6. Time dependence of the fractions of particles having amorphous (solid line), MRCO (dash-dot line), and bcc (dotted line) neighborhoods. Note the dominance of the amorphous structure at early times.

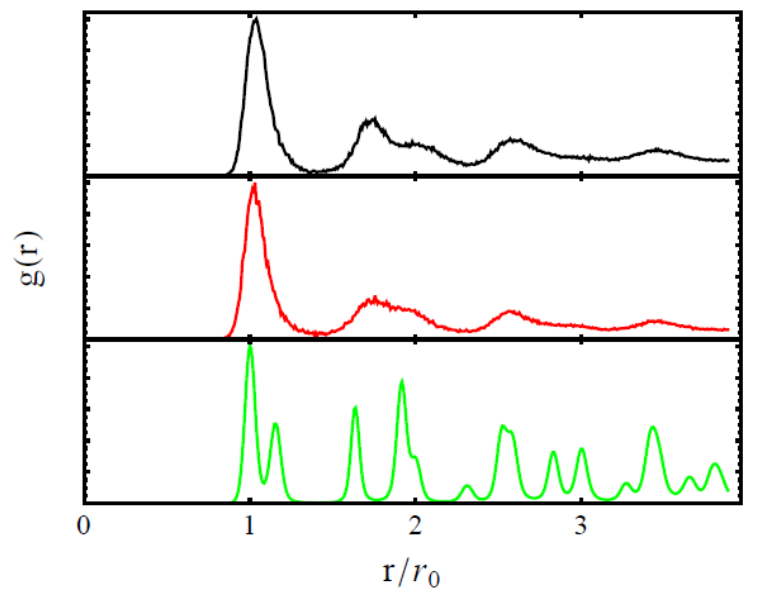

Fig. 7. Radial distribution functions (RDFs), $g(r)$, characterizing the amorphous (top), MRCO (middle), and bcc (bottom) structures. Note the closeness of the RDFs for the first two. The RDF for MRCO is in a good agreement with that of the bcc-like precursor observed in colloidal systems [37].

liquidlike, whereas $\bar{q}_{6}>0.4$ is bcc crystalline. Assigning red, white, and green colors to the respective particles, they visualized the spatiotemporal evolution of the undercooled liquid [40]. We have adopted a similar, but slightly different procedure by Tan et al. [37] in analyzing the PFC simulations. We

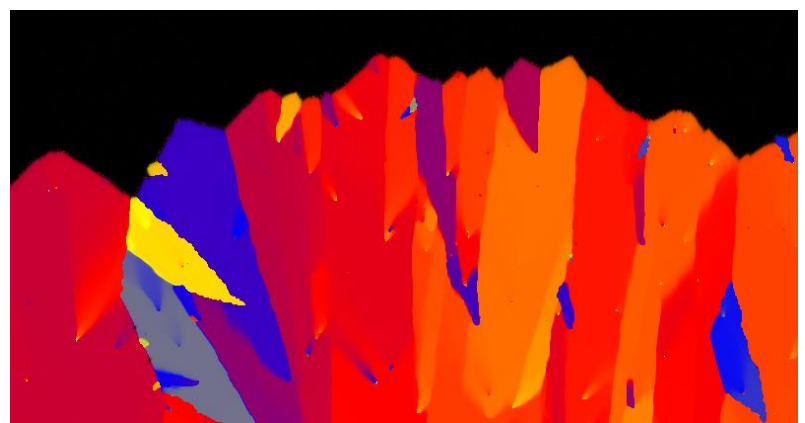

Fig. 8. Orientation map from a phase-field model of spherulitic solidification relying on an orientation field [40, 41]. The formation of orientation defects at the solid-liquid interface is shown that initiate new grains. Different colors correspond to different crystallographic orientations. Sharp changes in color stand for grain boundaries (coherent dislocation lines), whereas the orientation defects can be interpreted as bundles of dislocations. The liquid domain is black. It can be viewed as a randomized version of the interface breakdown proposed by Oldfield et al. [42]. show the respective MRCO and bcc regions in the first row of Fig. 5. The overall similarity between the BD and PFC simulations is fairly reasonable: in both cases, appearance of MRCO regions precedes the formation of the bcc crystals, which evolve inside/in connection with the MRCO domains. Remarkably, however, in the PFC simulation a disordered (amorphous) solid appears first (see Fig. 6), that is structurally similar to the liquid. Apparently, the white domains in the first row of Fig. 3, can be divided into two types of sub-domains, one containing MRCO and the other, a solid state of liquidlike structure. We note that the latter has not been reported in BD simulations. One may speculate that the amorphous solid and the liquid regions can be distinguished via investigating time correlations in the local structure, which has yet to be performed for the BD simulations. The radial distribution functions (RDFs) for the bcc, MRCO, and amorphous structures are displayed in Fig. 7. The RDFs predicted by the PFC model are close to those from molecular dynamics simulations for undercooled and crystalline $\mathrm{Fe}[41,42]$, whereas the RDFs of the amorphous and MRCO structures resemble closely to the RDF of the bcc-like precursor observed in experiments on colloids [37].

\subsection{Quest for atomistic modeling of Growth Front Nucleation}

Growth Front Nucleation (GFN: formation of new grains at the propagating solidification front) has been identified as the mechanism by which complex polycrystalline growth forms appear [23, 24, 43]. This phenomenon has been successfully modeled by phase-field methods relying on orientation field that describe the local crystallographic orientation. In this approach, the formation of new grains happens either by quenching in orientational defects into the crystal [23, 24, 44] (see Fig. 8 ), or by branching in the directions of low grain-boundary energies $[24,43]$. This approach, relying on coarse grained fields, became fairly successful in capturing rather complex structures $[23,24,43,45]$. Yet it is desirable to clarify the microscopic background of the GFN processes. Here, we address the formation of new grains at the solid-liquid interface at high undercoolings or supersaturations within the framework of the PFC and HPFC models [13].

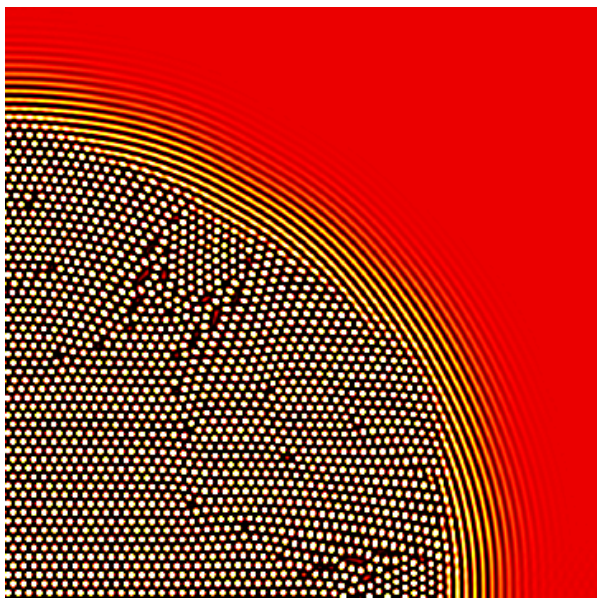

Fig. 9. Density waves, the formation of defects and new crystallographic orientations beyond the linear stability limit of the liquid in the original PFC model. Here $\alpha=0, \psi_{0}=-0.45, \varepsilon=0.75$, whereas $\psi_{\mathrm{c}}$ $=-0.5$. 

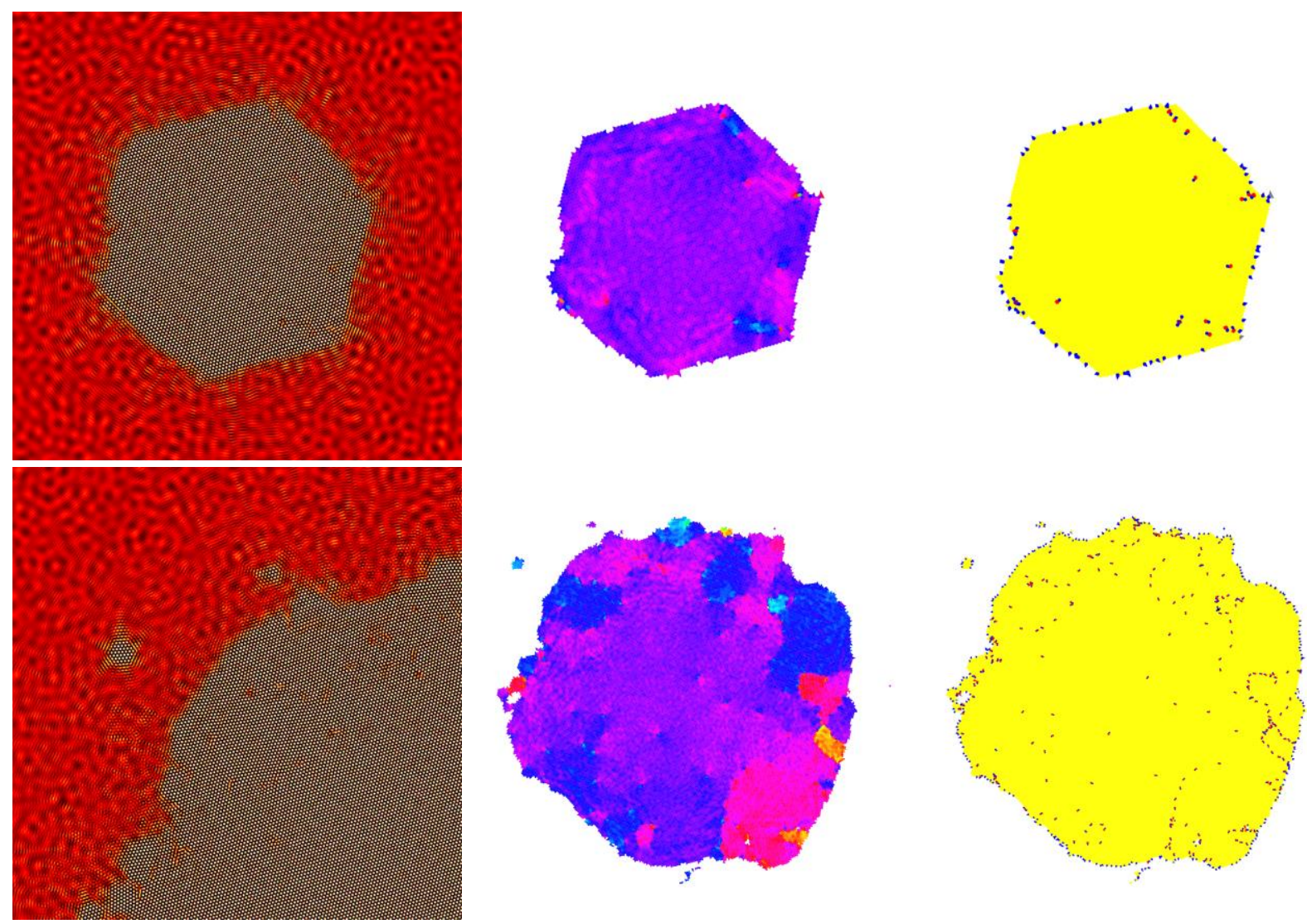

Fig. 10. Polycrystalline growth in the HPFC model in the metastable liquid regime in the presence of momentum noise. The simulation was performed on a $4096^{2}$ rectangular grid. The snapshots were taken at dimensionless time $t=1500$ (upper row; central $1024^{2}$ section are shown) and 3000 (lower row; $1024^{2}, 2048^{2}$ and $2048^{2}$ sections are shown). Left: number density map. Center: orientation map (different colors correspond to different orientations). Right: Voronoi polyhedral map showing the dislocations/grain boundaries (the same coloring is used as in Fig. 1: dislocations are represented by blue-red pairs). Two types of GFN can be seen: (a) the formation of dislocations (probably misfit dislocations) at the corners and at the center of the edges of the initially hexagonal crystal (see upper row), and (b) nucleation of differently oriented crystallites near the solid-liquid interface (emerging from the interference of the density waves emanating from the rough surface of the crystal, see lower row). Process (a) leads to smaller deviations from the orientation of the seed that started freezing and seem to be present from the very beginning, whereas process (b) that takes place at later stages yields orientations that are apparently independent from the original one.

Our first attempts to model GFN were made years back [25] using the original PFC model. Without applying noise, while going to supersaturations beyond the linear stability limit of the homogeneous liquid $\left[\psi_{\mathrm{c}}=-(\varepsilon / 3)^{1 / 2}\right]$, we have observed that a crystal seed developed into an ordered polycrystalline structure shown in Fig. 9 during growth. When going beyond the stability limit, the solid-liquid interface increasingly extends, and the growing crystal is surrounded by concentric density waves, which initiate crystallization accordingly. In six directions these waves help the growth of the original crystal, whereas in other directions a large number of defects form and new orientations appear that fit better to the density waves (Fig. 9). This could be viewed as an elementary process for GFN. Unfortunately, in this regime the liquid is unstable: when noise is added to the EOM, all these phenomena are suppressed by an explosive crystallization taking place everywhere. Inside the stable / metastable regime, we were unable to observe the GFN phenomenon.

In the case of the recently developed HPFC model [13], we were more successful in this respect. Starting with a small seed, we were able to grow polycrystalline domains as illustrated in Fig. 10. We used materials properties given in Ref. [13]. Other conditions were, $\varepsilon=-0.0923$, and the initial density $\psi_{0}=\psi_{\mathrm{c}}-$
$0.11 \Delta$, where $\Delta=\psi_{\mathrm{c}}-\psi_{\mathrm{L}}=0.0028$, while the respective densities at the liquidus line and at the stability limit are $\psi_{\mathrm{L}}=$ 0.1982 and $\psi_{c}=-0.1754$, respectively. Eqs. (5) and (6) were solved on a $4096^{2}$ rectangular grid with periodic boundary conditions on all sides. To analyze the local structure, we used a complex order parameter $g_{6}=\sum_{j} \exp \left\{-6 i \theta_{j}\right\}$, where the summation is performed for the nearest neighbors, and $\theta_{j}$ is the angle of the $j^{\text {th }}$ neighbor in the laboratory frame. The magnitude of $g_{6}$ represents the degree of order in the neighborhood, whereas its phase shows the local orientation. We have also performed Voronoi polyhedral analysis of the growing crystal using the same coloring as in the case of heteroepitaxy.

The simulation was performed in the presence of noise in a regime, where the liquid is metastable with respect to the fluctuations. The crystal seed starts to grow as a single crystal, but gradually new orientations appear via two mechanisms of GFN: (1) Dislocations enter the hexagonal crystal initially at the corners and at the center of the edges, later at cusps of the solid-liquid interface. These are probably misfit dislocations, emerging from the stress field of the growing crystal, however, further analysis is needed to prove this. (2) Small crystallites nucleate in the neighborhood of the solid-liquid interface, which apparently originate from the interference of the density 
waves emanating from the solid-liquid interface. Work is underway to quantify these phenomena.

Finally, it remains an intriguing question why the HPFC model recovers GFN at large driving forces, while the PFC supplemented with diffusive dynamics does not. A possible explanation can be that, in the latter case, a fast growth mode characterized by a broad interface occurs at large driving forces [46], in which healing of the dislocations can be relatively easy, avoiding thus the formation of dislocations at the perimeter of the growing crystal, preventing thus GFN.

\section{Summary}

We have addressed three problems related to crystallization in undercooled liquids within the framework of a simple dynamical density functional theory, the PFC model, and its hydrodynamic extension (HPFC):

(i) We investigated the misfit dependence of the critical thickness in heteroepitaxy, where we found that the PFC simulations fit better to the People-Bean relationship then to the Matthews-Blakeslee model, and that different slopes were predicted by the PFC model for compressive and tensile stresses.

(ii) We have studied the structural features of the amorphous precursor assisting crystal nucleation the PFC theory predicts at high undercoolings. An analysis in terms of bond order parameters, implies that the structure of the amorphous precursor is close to that of the Lennard-Jones liquid, whereas the respective radial distribution function resembles closely those from molecular dynamics for undercooled Fe.

(iii) We attempted to describe the formation of new grains at the growth front (GFN) within continuum theory (PFC and HPFC). While we were unable to find GFN, when using the PFC model with diffusive dynamics, we have observed microscale processes in the HPFC model leading to GFN: (a) dislocations enter into the crystal initially at the tips and at the center of the faces, later at the cusps of the solid-liquid interface (probably due to stresses building up in the growing crystallite), and (b) new grains nucleate close to the solid-liquid interface owing to the interference of density waves ahead of the rough solid-liquid front.

\section{Acknowledgments}

This work has been supported by National Agency for Research, Development, and Innovation (NKFIH), Hungary under contract No. OTKA-K-115959, by the EU FP7 Collaborative Project "EXOMET" (contract no. NMP-LA-2012-280421, co-funded by ESA), and by the ESA MAP/PECS projects "MAGNEPHAS III" (ESTEC Contract No. 40000110756/11/ NL/KML) and "GRADECET" (ESTEC Contract No. 40000 110759/11/ NL/KML).

\section{References:}

[1] K.R. Elder, M. Katakowski, M. Haataja, M. Grant, Phys. Rev. Lett. 88 (2002) 245701.

[2] K.R. Elder, N. Provatas, J. Berry, P. Stefanovic, M. Grant, Phys. Rev. B 75 (2007) 064107.

[3] K.-A. Wu, A. Adland, A. Karma, Phys. Rev. E 81 (2010) 061601.

[4] H. Emmerich, H. Löwen, R. Wittkowski, T, Gruhn, G.I. Tóth, G. Tegze, L. Gránásy, Adv. Phys. 61 (2012) 665. For a review on PFC modeling of nucleation see Sections 4 and 5.1.1.
[5] R. Backofen, A. Voigt, J. Phys.: Condens . Matter 22 (2010) 364101 .

[6] G.I. Tóth, T. Pusztai, G. Tegze, G. Tóth, L. Gránásy, Phys. Rev. Lett. 107 (2011) 175702.

[7] G.I. Tóth, T. Pusztai, G. Tegze, L. Gránásy, Phys. Rev. Lett. 108 (2012) 025502

[8] Gránásy, F. Podmaniczky, G.I. Tóth, G. Tegze, T. Pusztai, Chem. Soc. Rev. 43 (2014) 2159.

[9] G. Tegze, L. Gránásy, G.I. Tóth, J. F. Douglas, T. Pusztai, Soft Matter 7 (2011) 1789.

[10] G.I. Tóth, G. Tegze, T. Pusztai, G. Tóth, L. Gránásy, J. Phys.: Condens. Matter 22 (2010) 364101.

[11] J. Berry, K.R. Elder, M. Grant, Phys. Rev. E 77 (2008) 061506.

[12] J. Berry, M. Grant, Phys. Rev. Lett. 106 (2011) 175702.

[13] G.I. Tóth, L. Gránásy, G. Tegze, J. Phys.: Condens. Matter 26 (2014) 055001.

[14] J.W. Matthews, A.E. Blakeslee, J. Cryst. Growth 27 (1974) 118.

[15] K.R. Elder, M. Grant, Phys. Rev. E 70 (2004) 051605.

[16] F. Podmaniczky, G.I. Tóth, G. Tegze, L. Gránásy, Metall. Mater. Trans. A 46 (2015) 4908.

[17] R. Backofen, A. Voigt, Eur. Phys. J. Special Topics, 223 (2014) 497.

[18] J.F. Lutsko, G. Nicolis, Phys. Rev. Lett. 96 (2006) 046102.

[19] T. Schilling, H.J. Schöpe, M. Oettel, G. Opletal, I. Snook, Phys. Rev. Lett. 105 (2010) 025701.

[20] H.J. Schöpe, G. Bryant, W. van Megen, Phys. Rev. Lett. 96 (2006) 175701

[21] T.H. Zhang, X.Y. Liu, J. Am. Chem. Soc. 129 (2007) 13520.

[22] T.H. Zhang, X.Y. Liu, J. Phys. Chem. B 111 (2007) 14001.

[23] L. Gránásy, T. Pusztai, T. Börzsönyi, J A. Warren, J.F. Douglas: Nature Mater. 3 (2004) 645.

[24] L. Gránásy, L. Rátkai, A. Szállás, B. Korbuly, G.I. Tóth, L. Környei, T. Pusztai, Metall. Mater. Trans. A 45 (2014) 1694.

[25] L. Gránásy, G. Tegze, unpublished result (2009).

[26] S. van Teeffelen, R. Backofen, A. Voigt, H. Löwen, Phys. Rev. E 79 (2009) 051404.

[27] T.V. Ramakrishnan, M. Yussouff, Phys. Rev. B 19 (1979) 2775.

[28] J.G. Ojalvo and J.M. Sancho, Noise in Spatially Extended Systems (Springer, New York, 1999), p. 175.

[29] B.Z. Shang, N.K. Voulgarakis. J.-W. Chu, J. Chem. Phys. 135 (2011) 044111.

[30] G. Tegze, G. Bansel, G.I. Tóth, T. Pusztai, Z. Fan, L. Gránásy, J. Comput. Phys. 228 (2009) 1612.

[31] R.J. Asaro, W.A. Tiller, Metall. Trans. 3 (1972) 1789.

[32] M. Grinfeld, Dokl. Akad. Nauk SSSR 290 (1986) 1358 [Sov. Phys. Dokl. 31 (1986) 831].

[33] Z.-F. Huang, K.R. Elder, Phys. Rev. Lett. 101 (2008) 158701.

[34] R. People, J.C. Bean, J. Appl. Phys. 47 (1985) 322.

[35] J.E. Ayers, Heteroepitaxy of Semiconductors: Theory, Growth, and Characterization (CRC Press, 2007).

[36] A. Fischer, H. Kühne, H. Richter, Phys. Rev. Lett. 73 (1994) 2712.

[37] P. Tan, N. Xu, L. Xu, Nature Phys. 10 (2014) 73.

[38] P.J. Steinhardt, D.R. Nelson, M. Ronchetti, Phys. Rev. B 28 (1983) 784

[39] W. Lechner, C. Dellago, J. Chem. Phys. 129 (2008) 114707.

[40] T. Kawasaki, H. Tanaka, Proc. Natl. Acad. Sci. (US) 107 (2010) 14037.

[41] Y. Shibuta, T. Suzuki, J. Chem. Phys. 129 (2008) 144102.

[42] V.V. Hoang, Nanothechnol. 20 (2009) 295703.

[43] L. Gránásy, T. Pusztai, G. Tegze, J.A. Warren, J.F. Douglas, Phys. Rev. E. 72 (2005) 011605.

[44] W. Oldfield, G.T. Geering, W.A. Tiller, The Soldification of Metals (Iron and Steel Inst. Publ., London, 1968) p. 110.

[45] N. Hendler, E. Mentovich, B. Korbuly, T. Pusztai, L. Gránásy, S. Richter, Nano Res. 8 (2015) 3630.

[46] G. Tegze, G. I. Tóth, L. Gránásy, Phys. Rev. Lett. 106 (2011) 195502. 